\title{
Time to pause ventricular tachycardia: the PAUSE-SCD trial
}

\author{
David F. Briceño ${ }^{1} \cdot$ Jorge Romero $^{1} \cdot$ Isabella Alviz ${ }^{1} \cdot$ Nicola Tarantino $^{1} \cdot$ Luigi Di Biase $^{1}$ (ID
}

Received: 9 September 2019 / Accepted: 11 September 2019 /Published online: 24 October 2019

(C) Springer Science+Business Media, LLC, part of Springer Nature 2019

Patients with structural heart disease (SHD) are at increased risk for ventricular arrhythmias (VA) and mortality. Albeit, implantable cardiac defibrillators (ICD) have been efficacious in improving survival, while recurrent ventricular tachycardia (VT) with subsequent ICD shocks reduce quality of life and is associated with increased mortality [1]. Antiarrhythmic drugs (AADs) have been of limited value given suboptimal efficacy and numerous side effects [1]. Conversely, catheter ablation (CA) has evolved as a useful tool to control VA and improve quality of life. Most VT randomized controlled trials (RCTs) comparing CA with medical therapy have been conducted in patients with ischemic cardiomyopathy (ICM), and although not powered to show an all-cause mortality benefit, they have reported substantial superiority of $\mathrm{CA}$ to standard medical therapy in reducing ICD interventions and VT recurrences $[2,3]$. In fact, the VANISH trial showed that CA reduced the composite primary outcome of death, VT storm, or appropriate ICD shock compared to patients receiving an escalation in AADs [4]. On the other hand, the role of CA of VT in patients with non ischemic cardiomyopathy (NICM) is variable and largely depends on the arrhythmogenic substrate. Furthermore, outcome data on mortality benefit of CA for VT in NICM are limited [5, 6]. Patients with NICM, which are highly prevalent in Asia, have not been previously enrolled in RCTs of VT ablation. Few observational studies have reported encouraging outcomes in this population. Tung et al. [7] reported, in a large international VT ablation study of 2000 patients, that CA of VT in patients with structural heart disease results in $70 \%$ freedom from VT recurrence similar for both ICM and NICM (72\% in ICM and 68\% in NICM), with an overall transplant and/or mortality rate of $15 \%$ at 1 year (same for ICM and NICM). Subsequently, our group analyzed a large-scale data of NICM patients from the 2003-2014

Luigi Di Biase

dibbia@gmail.com

1 Montefiore-Einstein Center for Heart and Vascular Care, Montefiore Medical Center, Albert Einstein College of Medicine, 111 East 210th Street, Bronx, NY 10467, USA
National Inpatient Sample databases [8]. A propensity score matched analysis was used to compare patients undergoing CA versus medical therapy of VT related to NICM and described the temporal trends in utilization and in-hospital outcomes of CA of VT in patients with NICM in the USA. Out of 133,529 patients hospitalized with the principal diagnosis of VT in NICM, 14,651 (11.0\%) underwent CA. After propensity score matching, in-hospital mortality occurred in 172 of $14,318(1.2 \%)$ patients in the CA group, compared with 297 of $14,156(2.1 \%)$ of patients undergoing medical therapy (adjusted OR, 0.53 ; 95\% CI, 0.43-0.66), which translates into a $47 \%$ relative reduction in all-cause mortality.

In this issue of the journal, Chen et al. [9]report the rational and trial design of a protocol envisioned to evaluate whether preemptive CA followed by ICD implantation in patients with SHD (LVEF < 50\%), including patients with NICM, and monomorphic VT results in improved clinical outcomes compared to ICD implantation with standard medical therapy alone. All patients randomized to $\mathrm{CA}$ will undergo a substrate-based strategy using the NAVX electroanatomic 3D mapping system (Ensite, Velocity or Precision, Abbott, Lake Forest, IL) and a substrate homogenization approach is encouraged. Patients will be randomized in a 1:1 fashion to two treatment arms: ICD with ablation and ICD with standard medical therapy alone (betablockers, AADs). A 50\% reduction in events from $\mathrm{CA}$ is projected with a $50 \%$ event rate in the control arm at 2 years; 120 patients were calculated to achieve $80 \%$ power to detect a true difference of this magnitude with a two-sided alpha error of 0.05 . Interestingly, a registry is planned for patients that refuse randomization to examine the natural history of CA outcomes in the absence of background ICD therapy.

Therefore, the trial will also provide information in regard to the "necessity" of ICD implantation following a successful ablation in this patient population. The protocol is exceedingly appealing considering that the role of $\mathrm{CA}$ as an adjunct and alternative to ICD implantation is not known in patients at risk for recurrent VT and sudden cardiac death (SCD). The two previous landmark trials (SMASH-VT and VTACH) [2, 3] with similar design evaluating prophylactic VT ablation were 
both conducted in patients with ischemic VT. As such, the role of this strategy in the setting of NICM is unknown, but considering the encouraging emerging ablation outcomes in these patients, a trial was long due. A major strength is the use of a substrate based ablation strategy rather than standard approach considering the improved long-term outcomes shown with the former [10]. Furthermore, advocating a substrate homogenization is critical, considering this is one of the only substrate strategies shown to be effective in both ICM and NICM [11, 12]. Despite these strengths, some limitations may confound results, such as the lack of a uniform control group based only on AADs as operators may be able to choose any medical therapy. The main limitation, which is understandable from the procedural and expertise perspective, is the lack of a pre-specified epicardial mapping and ablation strategy, which is encouraged but not mandated. Unfortunately, this limitation may bias or even confound results, particularly considering that most substrate in NICM is actually epicardial or mid-myocardial. Similarly, several studies have shown the beneficial effect of epicardial ablation even in ICM [13]. This being said, the effort is enormous and results are eagerly awaited. Hopefully, this effort is another path to finally pause VT.

\section{Compliance with ethical standards}

Conflict of interest Dr. Di Biase is a consultant for Biosense Webster, Stereotaxis, Boston Scientific and Abbott Medical. Dr. Di Biase received speaker honoraria/travel support from Medtronic, Atricure, EPiEP, and Biotronik. The remaining authors have no disclosures.

\section{References}

1. Zipes DP, Camm AJ, Borggrefe M, et al. ACC/AHA/ESC 2006 guidelines for management of patients with ventricular arrhythmias and the prevention of sudden cardiac death: a report of the American College of Cardiology/American Heart Association Task Force and the European Society of Cardiology Committee for Practice Guidelines (Writing Committee to Develop Guidelines for Management of Patients With Ventricular Arrhythmias and the Prevention of Sudden Cardiac Death). J Am Coll Cardiol. 2006;48:e247-346.

2. Reddy VY, Reynolds MR, Neuzil P, Richardson AW, Taborsky M, Jongnarangsin $\mathrm{K}$, et al. Prophylactic catheter ablation for the prevention of defibrillator therapy. N Engl J Med. 2007;357:2657-65.
3. Kuck KH, Schaumann A, Eckardt L, Willems S, Ventura R, Delacrétaz E, et al. Catheter ablation of stable ventricular tachycardia before defibrillator implantation in patients with coronary heart disease (VTACH): a multicentre randomised controlled trial. Lancet. 2010;375:31-40.

4. Sapp JL, Wells GA, Parkash R, Stevenson WG, Blier L, Sarrazin $\mathrm{JF}$, et al. Ventricular tachycardia ablation versus escalation of antiarrhythmic drugs. N Engl J Med. 2016;375:111-21.

5. Muser D, Santangeli P, Castro SA, et al. Long-term outcome after catheter ablation of ventricular tachycardia in patients with nonischemic dilated cardiomyopathy. Circ Arrhythm Electrophysiol. 2016;9.

6. Bai R, Di Biase L, Shivkumar K, et al. Ablation of ventricular arrhythmias in arrhythmogenic right ventricular dysplasia/cardiomyopathy: arrhythmia-free survival after endo-epicardial substrate based mapping and ablation. Circ Arrhythm Electrophysiol. 2011;4:478-85.

7. Tung R, Vaseghi M, Frankel DS, Vergara P, Di Biase L, Nagashima $\mathrm{K}$, et al. Freedom from recurrent ventricular tachycardia after catheter ablation is associated with improved survival in patients with structural heart disease: an international VT ablation center collaborative group study. Heart Rhythm. 2015;12:1997-2007.

8. Briceno DF, Gupta T, Romero J, et al. Catheter ablation of ventricular tachycardia in nonischemic cardiomyopathy: a propensity score-matched analysis of in-hospital outcomes in the United States. J Cardiovasc Electrophysiol. 2018;29:771-9.

9. Chen M, Wu S, Yao Y, Jiang J, Jiang C, Xue Y, et al. on behalf of the PAUSE-SCD investigators. Pan-Asia United States PrEvention of Sudden Cardiac Death Catheter Ablation Trial (PAUSE-SCD): rationale and study design. J Interv Card Electrophysiol. 2019. https://doi.org/10.1007/s10840-019-00535-w.

10. Briceno DF, Romero J, Villablanca PA, et al. Long-term outcomes of different ablation strategies for ventricular tachycardia in patients with structural heart disease: systematic review and meta-analysis. Europace. 2018;20:104-15.

11. Gokoglan Y, Mohanty S, Gianni C, et al. Scar homogenization versus limited-substrate ablation in patients with nonischemic cardiomyopathy and ventricular tachycardia. J Am Coll Cardiol. 2016;68:1990-8.

12. Di Biase L, Burkhardt JD, Lakkireddy D, et al. Ablation of stable VTs versus substrate ablation in ischemic cardiomyopathy: the VISTA randomized multicenter trial. J Am Coll Cardiol. 2015;66: 2872-82.

13. Romero J, Cerrud-Rodriguez RC, Di Biase L, et al. Combined endocardial-Epicardial versus endocardial catheter ablation alone for ventricular tachycardia in structural heart disease: a systematic review and meta-analysis. JACC Clin Electrophysiol. 2019;5:13-24.

Publisher's note Springer Nature remains neutral with regard to jurisdictional claims in published maps and institutional affiliations. 